Research Article

\title{
The Active Fractional Order Control for Maglev Suspension System
}

\author{
Peichang Yu, Jie Li, and Jinhui Li \\ College of Mechatronics Engineering and Automation, National University of Defense Technology, Changsha 410073, China \\ Correspondence should be addressed to Jie Li; jieli@nudt.edu.cn
}

Received 22 October 2014; Revised 4 March 2015; Accepted 5 March 2015

Academic Editor: Andrzej Swierniak

Copyright ( 2015 Peichang Yu et al. This is an open access article distributed under the Creative Commons Attribution License, which permits unrestricted use, distribution, and reproduction in any medium, provided the original work is properly cited.

\begin{abstract}
Maglev suspension system is the core part of maglev train. In the practical application, the load uncertainties, inherent nonlinearity, and misalignment between sensors and actuators are the main issues that should be solved carefully. In order to design a suitable controller, the attention is paid to the fractional order controller. Firstly, the mathematical model of a single electromagnetic suspension unit is derived. Then, considering the limitation of the traditional $P D$ controller adaptation, the fractional order controller is developed to obtain more excellent suspension specifications and robust performance. In reality, the nonlinearity affects the structure and the precision of the model after linearization, which will degrade the dynamic performance. So, a fractional order controller is addressed to eliminate the disturbance by adjusting the parameters which are added by the fractional order controller. Furthermore, the controller based on $L Q R$ is employed to compare with the fractional order controller. Finally, the performance of them is discussed by simulation. The results illustrated the validity of the fractional order controller.
\end{abstract}

\section{Introduction}

The developing of maglev train is under a rapid speed around the world. Thanks to no physic contact with guide-way, the maglev system gets the advantage of lower noise, less costly maintenance, less exhaust fumes emission, and so on, which is suitable for the urban transportation. Until now, two commercial maglev routes and several test routes have been built around the world.

Suspension control is one of the core technologies in maglev train $[1,2]$. The practical model of suspension system is a nonlinear and inherent unstable system due to the fact that the electromagnetic force produced by a constant current is inversely proportional to the square of the levitation gap. Besides, the parameters of the levitation system are varying with the difference of the operating condition. So, it is hard to choose a practicable control strategy to design an ideal controller that can keep satisfied dynamic performance when the train is running under the actual environment. Considering the complicated working situation, many researchers have investigated many new controllers with different theories for the maglev suspension system such as adaptive control [3], nonlinear control [4], fuzzy control [5], and robust control [6].
The quick development of fractional calculus attracts extensive attentions due to its nonlinear feature and unique memory characteristic. In electricity, properties between resistance and capacitance are intermediated by a concept of fractance [7]. Fractional calculus has been applied in the modeling and control of various kinds of physical systems and many real systems are modeled or fitted by fractional order systems. So, in recent years, with the progress of fractional order integral theory, many papers have discussed the design and application of controller design based on fractional order theory.

Fractional order $P D$ controller attracts increasing attention because of the higher freedom degree provided [8-11]. In paper [8] a TID controller is proposed; this control scheme can improve the robustness of system against disturbances. In paper [9], Oustaloup discussed the CRONE controller. In paper [12], Podlubny focused on the $P I^{\lambda} D^{\mu}$ controller; this method is the milestone of the FOC; it increased two parameters for the controller, so, with the $P I^{\lambda} D^{\mu}$ controller, the system can get desired performance. Since then, many works have been done to apply these new methods to different nonlinear systems. 


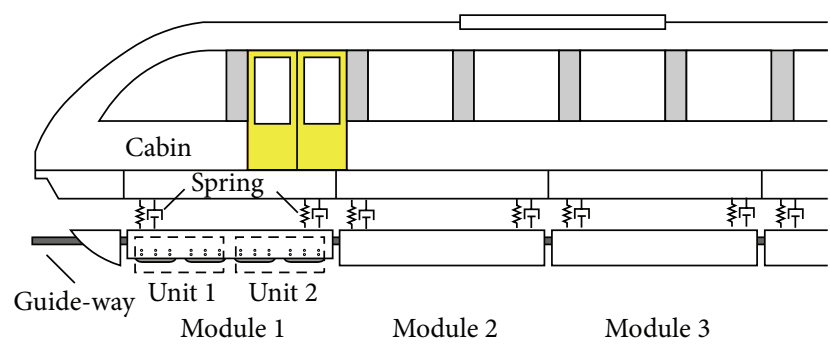

FIGURE 1: Side view of CMS04.

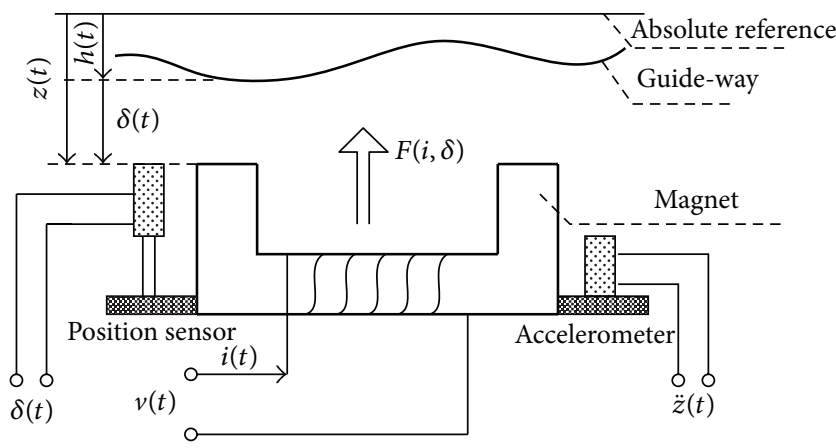

FIGURE 2: Electromagnet-track configuration of a single unit.

The maglev train, as a typical nonlinear system, is an ideal model for fractional order controller design. This essay paid attention to discussing the usage of fractional order control theory and application of the $P D^{\mu}$ controller on solving the system inherent nonlinearity and parameters uncertain problems of the suspension system. The rest of the paper is organized as follows. The mathematical model of the basic maglev suspension system is developed in Section 2. In Section 3, the design theory and procedure of $P D^{\mu}$ controller based on the simplified model are discussed. In Section 4, simulation is proposed, and we compared the performance with controller based on $L Q R$. Finally, conclusions are presented in Section 5.

\section{Modeling}

A whole maglev train includes the air springs, actively controlled electromagnets, and the cabin. An overall view of the maglev vehicle is shown in Figure 1. The maglev train is made up of ten levitation modules, and each module can be decomposed into two single electromagnetic suspension units which are shown in Figure 2. To derive the dynamic model of the single electromagnetic suspension unit, from the opinion of engineering, several hypotheses were made. Firstly, it is assumed that the mass of cabin is transferred to the bogie only in the longitudinal direction. Secondly, neglecting the flux leakage of the electromagnetic due to the operating levitation gap is $9 \mathrm{~mm}$. Finally, considering the working gap and engineering analysis, the magnetic resistances of the ferrite core and rail are neglected; namely, the magnetic potential falls on the air gap evenly.
The fundamental principle of the electromagnet suspension system (shown in Figure 2) is described as the following: according to the desired suspension gap and status information gotten by position sensor and accelerometer, the controller adjusts the current supply so that the suspension system can produce suitable force $F$ that can overcome the gravitation and keep the system stable in the expected operating position. A single electromagnet suspension unit can be simplified as shown in Figure 2 .

In Figure 2, $m$ is the total mass of suspension system. $N$ denotes the number of turns of a single electromagnet. $A$ is the area of the magnetic pole. $R$ is the magnetic resistance. $\mu_{0}$ is the magnetic permeability of atmosphere. $g$ is the gravity acceleration. The magnetic inductance is defined as $L . F$ is the force produced by electromagnetic for suspension. $\delta$ is the measured position value from position sensor. $\ddot{z}$ means the value of measured acceleration from accelerometers. The voltage supplied to magnetic suspension system is expressed by $v$. The symbol $i$ is the current of magnetic suspension system. $B$ is the flux density of the gap. $f_{d}(t)$ is the disturbance of the load.

There are three main relations in the suspension system; there are voltage $(v)$ to current $(i)$, acceleration $(\ddot{z})$ to electromagnetic force $(F)$, and current $(i)$ to electromagnetic force $(F)$. Based on the electromagnetic theory and dynamics analysis of electromagnet [1], the following equations can be built:

$$
\begin{gathered}
v(t)=R i(t)+\frac{d(L(t) i(t))}{d t}, \\
m \ddot{z}(t)=m g-F(t, \delta)+f(t), \\
z(t)=\delta(t)+h(t),
\end{gathered}
$$

where $F(i, \delta)=B^{2} A / \mu_{0}, L(t)=\mu_{0} N^{2} A / 2 \delta(t)$, and $B(t)=$ $\mu_{0} N i(t) / 2 \delta(t)$.

There is a need to pay attention to the fact that this model did not put the guide-way irregularities into consideration. So, with $h(t)=0, z(t)$ is equal to $\delta(t)$. From (1), it is obvious that the system is internally nonlinear, and it will be discussed later. Furthermore, simplifying the above equations, the following equation can be deduced:

$$
\begin{gathered}
v(t)=R i(t)+\frac{\mu_{0} N^{2} A}{2} \frac{\dot{i}(t)}{\delta(t)}-\frac{\mu_{0} N^{2} A i(t)}{2} \frac{\dot{\delta}(t)}{[\delta(t)]^{2}}, \\
m \ddot{z}(t)=m g-F(i, \delta)+f(t), \\
F(i, \delta)=\frac{\mu_{0} N^{2} A}{4}\left[\frac{i(t)}{\delta(t)}\right]^{2} .
\end{gathered}
$$

From (2), it is obvious that electromagnetic force $F$ is dependent on the current $i$ and gap $\delta$; the relationship between them is nonlinear. That means there is nonlinear relationship between them and it is the source of the system nonlinearity.

Traditionally, the model is linearized in the general working point $\left(i_{0}, \delta_{0}\right)$, although this will bring the loss of precision, but it is still an effective way to analyse the main 
feature of the system. Certainly, it is a good idea to design the controller based on the nonlinear model, but it is necessary to consider the difficulty brought for the model building and difficulty of controller designing.

The linearized system can be described as

$$
\begin{gathered}
\Delta \dot{i}(t)=\frac{k_{z}}{k_{i}} \Delta \dot{\delta}(t)-\frac{R}{L_{0}} \Delta i(t)+\frac{1}{L_{0}} \Delta v(t), \\
m \Delta \ddot{z}(t)=\Delta F(i, \delta)+f(t), \\
\Delta F(i, t)=-k_{i} \Delta i(t)+k_{z} \Delta \delta(t), \\
\Delta z(t)=\Delta \delta(t)+\Delta h(t)
\end{gathered}
$$

where $k_{i}=-\mu_{0} N A i_{0} / 2 \delta_{0}^{2}, k_{z}=-\mu_{0} N A i_{0}^{2} / 2 \delta_{0}^{3}$. Replacing $\Delta z(t), \Delta i(t)$, and $\Delta v(t)$ with $z, i$, and $v$ and defining that $U=v(t), Y=\delta(t)$, and $X=\left[\begin{array}{lll}\delta(t) & \dot{z}(t) & i(t)\end{array}\right]^{T}$, the state-space representation of the above equation is

$$
\begin{gathered}
\dot{X}=A^{\prime} X+B^{\prime} U+f, \\
Y=C^{\prime} X,
\end{gathered}
$$

where

$$
\begin{gathered}
A^{\prime}=\left[\begin{array}{ccc}
0 & 1 & 0 \\
\frac{k_{z}}{m} & 0 & -\frac{k_{i}}{m} \\
0 & \frac{k_{z}}{k_{i}} & -\frac{R}{L_{0}}
\end{array}\right], \\
B^{\prime}=\left[\begin{array}{c}
0 \\
0 \\
-\frac{1}{L_{0}}
\end{array}\right], \quad C^{\prime}=\left[\begin{array}{lll}
1 & 0 & 0
\end{array}\right],
\end{gathered}
$$

where $f$ includes the disturbance of $f_{d}(t)$ from track, cabin, and other environment factors. From (4), it is obvious that the value of parameters is changing according to the working position $\delta_{0}$. Furthermore, the disturbance $f(t)$ is varying with the working environment at the same time. Facing so much uncertainty, as a good controller for the suspension system, it not only should keep the system stable in various working positions, but also has to reject the disturbance from environment.

\section{Procedure of Controller Design}

In this section, firstly, the classical PID controller design based on the method of $L Q R$ is recommended [13]. Then, the procedure of fractional order $\left(P D^{u}\right)$ controller design is introduced based on the principle of designing a fractional order controller and the suspension system model built in Section 2 .

3.1. Classical PD Controller Based on LQR. There are many ways to design classical PID controllers. This part focused on the design of $L Q R$ controller.
For a state-space equation,

$$
\begin{gathered}
\dot{x}(t)=A^{\prime \prime}(t) x(t)+B^{\prime \prime}(t) u(t), \\
y=C^{\prime \prime}(t) x(t) .
\end{gathered}
$$

$x(t)$ is a $n \times 1$ state matrix. $u(t)$ is a $m \times 1$ control matrix. $y(t)$ is a $l \times 1$ output matrix. $A^{\prime \prime}(t), B^{\prime \prime}(t)$, and $C^{\prime \prime}(t)$ are $n \times n, n \times m$, and $l \times n$ time varying matrices. The quadratic performance index of the equation can be expressed as

$$
\begin{aligned}
J= & \frac{1}{2} \int_{0}^{t_{f}}\left[x^{T}(t) Q x(t)+u^{T}(t) R u(t)\right] d t \\
& +\frac{1}{2} x^{T}\left(t_{f}\right) M x\left(t_{f}\right),
\end{aligned}
$$

where $Q, M$ are nonnegative definite matrix, $Q, R$ are weighting matrix of state-variable and input-variable separately, $R$ is positive definite matrix, $t_{f}$ is a fixed time, and $x\left(t_{f}\right)$ is free. The aim of the method is to be sure that the quadratic performance index $J$ should be the smallest, which means, in the process of control, the error and the consumption of energy are the least. The feedback control value is

$$
u(t)=-K x(t)
$$

Firstly, a Hamilton function is established:

$$
\begin{aligned}
H(t)= & \frac{1}{2} \int_{0}^{t_{f}}\left[x^{T}(t) Q x(t)+u^{T}(t) R u(t)\right] d t \\
& +\lambda^{T}(t)\left[A^{\prime \prime}(t) x(t)+B^{\prime \prime}(t) u(t)\right] .
\end{aligned}
$$

Then, making a derivation to $H(t)$, the smallest value can be calculated as

$$
\frac{\partial H(t)}{\partial u(t)}=-R u(t)+B^{\prime \prime T}(t) \lambda(t)=0 .
$$

Obviously

$$
u(t)=R^{-1} B^{\prime \prime T}(t) \lambda(t) .
$$

Following the above steps, a classical PID controller can be designed with desired performance.

3.2. Fractional Order PD Controller Design. Define a system transfer function $G(s)$ and its fractional order $P D$ controller $C(s)$ and $G_{-} o(s)$ is the open-loop transfer function. For the purpose of designing a fractional order $P D$ controller, there are three specifications needed to be met [14-16].

(a) Phase margin specification:

$$
\arg \left(G_{-} o\left(j \omega_{c}\right)\right)=-\pi+\phi_{m} .
$$

$\omega_{c}$ is the gain crossover frequency interested, $\phi_{m}$ is the phase margin, and $G_{-} o(j \omega)$ is the open-loop transfer function of the system. 
(b) Flat phase specification:

$$
d\left[\arg \left(G_{-} o(j \omega)\right)\right] /\left.d \omega\right|_{\omega_{c}}=0 .
$$

The phase Bode is flat at the gain crossover frequency. It means that the system is insensitive to the gain changes in the crossover frequency.

(c) Gain crossover frequency specification:

$$
\left|G_{-} o\left(j \omega_{c}\right)\right|=1 \text {. }
$$

3.2.1. Preliminary Knowledge. For a fractional order controller

$$
C(s)=K_{p}\left(1+K_{d} s^{\mu}\right)
$$

where $0<\mu$ and $K_{p}$ and $K_{d}$ are the relevant parameters needed to be calculated. The phase and gain of the controller can be expressed as

$$
\begin{gathered}
C(j \omega)=K_{p}\left(1+K_{d} \omega^{\mu} \cos \frac{\pi \mu}{2}+j K_{d} \omega^{\mu} \sin \frac{\pi \mu}{2}\right), \\
\arg (C(j \omega))=\arctan \frac{K_{d} \omega^{\mu} \sin (\pi \mu / 2)}{1+K_{d} \omega^{\mu} \cos (\pi \mu / 2)}, \\
|C(j \omega)|=K_{p} \sqrt{\left(K_{d} \omega^{\mu} \cos \frac{\pi \mu}{2}+1\right)^{2}+\left(K_{d} \omega^{\mu} \sin \frac{\pi \mu}{2}\right)^{2}} .
\end{gathered}
$$

According to (4), the suspension system transform function is given as

$$
G(s)=\frac{\delta(s)}{u(s)}=\frac{k_{i} / m L_{0}}{s^{3}+\left(R / L_{0}\right) s^{2}+k_{i} k_{p 1} / m L_{0}-k_{z} R / m L_{0}}
$$

Assuming $b_{0}=k_{i} / m L_{0}, a_{2}=R / L_{0}, a_{0}=k_{i} k_{p 1} / m L_{0}-$ $k_{z} R / m L_{0}$. Then it is similar to $C(s)$ that the phase and gain of $G(s)$ are

$$
\begin{aligned}
G(s)= & \frac{k_{i} / m L_{0}}{s^{3}+\left(R / L_{0}\right) s^{2}+k_{i} k_{p 1} / m L_{0}-k_{z} R / m L_{0}} \\
= & \frac{b_{0}}{s^{3}+a_{2} s^{2}+a_{0}}, \\
G(j \omega)= & \frac{b_{0}}{(j \omega)^{3}+a_{2}(j \omega)^{2}+a_{0}}=\frac{b_{0}}{-j \omega^{3}+a_{0}-a_{2} \omega^{2}}, \\
& \arg (G(j \omega))=\arctan \frac{\omega^{3}}{a_{0}-a_{2} \omega^{2}}, \\
& |G(j \omega)|=\frac{b_{0}}{\sqrt{\omega^{6}+\left(a_{0}-a_{2} \omega^{2}\right)^{2}}} .
\end{aligned}
$$




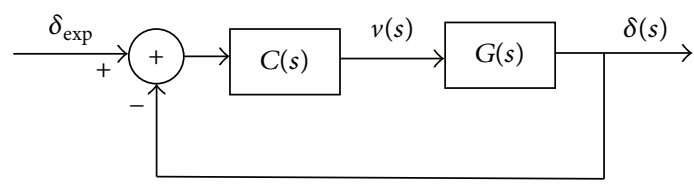

FIgURE 3: The diagram of closed-loop system.

where the parameters in (25) can be evaluated by the following equations:

$$
\begin{gathered}
\omega_{k}^{\prime}=\omega_{b}\left(\frac{\omega_{h}}{\omega_{b}}\right)^{(k+N+(1 / 2)(1-\mu)) /(2 N+1)}, \\
\omega_{k}=\omega_{b}\left(\frac{\omega_{h}}{\omega_{b}}\right)^{(k+N+(1 / 2)(1+\mu)) /(2 N+1)}, \\
K=\left(\frac{\omega_{h}}{\omega_{b}}\right)^{-\mu / 2} \prod_{k=-N}^{N} \frac{\omega_{k}}{\omega_{k}^{\prime}} .
\end{gathered}
$$

By using the Oustaloup Recursive Algorithm, a lot of convenience is brought.

\section{Simulation and Analysis}

4.1. Controller Designs. In order to discuss the fractional order $P D$ controller design based on Section 3, this section investigated the performance of fractional order $P D$ controller by simulation.

This paper chose a single standard maglev bogie as the model for study. The values of the basic parameters are listed in Table 1.

In practical, the input of the closed-loop system is the expected gap $\delta_{\exp }$ of the electromagnet. So the block diagram of the system can be shown in Figure 3.

By putting these parameters in the model, the mathematical model of suspension system in the working position $\delta_{0}$ can be expressed as

$$
\begin{gathered}
{\left[\begin{array}{c}
\dot{\delta}(t) \\
\ddot{z}(t) \\
\dot{i}(t)
\end{array}\right]=\left[\begin{array}{ccc}
0 & 1 & 0 \\
-2356 & 0 & 0.8 \\
0 & 2941 & 3.1
\end{array}\right]\left[\begin{array}{c}
\delta(t) \\
\dot{z}(t) \\
i(t)
\end{array}\right]+\left[\begin{array}{c}
0 \\
0 \\
5.6
\end{array}\right] v(t),} \\
y(t)=\delta(t)
\end{gathered}
$$

and the transfer function of the system can be expressed as

$$
G(s)=\frac{-4.525}{s^{3}+3.107 s^{2}-7320} .
$$

The $L Q R$ controller and fractional order controller can be designed according to Section 3.

The controller based on $L Q R$, by choosing $t f=+\infty$, $R=5$, and $Q=\left[\begin{array}{ccc}1 & 0 & 0 \\ 0 & 1 & 0 \\ 0 & 0 & 1\end{array}\right]$, and the feedback matrix can be calculated: $K=\left[\begin{array}{lll}-24869 & -210 & 7\end{array}\right]$. Certainly, when using the parameters, there is a need to do some fine adjustments.
TABLE 1: Parameters of the levitation system.

\begin{tabular}{lcc}
\hline Variables & Value & Unit \\
\hline$m$ & 653 & {$[\mathrm{~kg}]$} \\
$N$ & 360 & {$[-]$} \\
$A$ & 0.0185 & {$\left[\mathrm{~m}^{2}\right]$} \\
$R$ & 0.5 & {$[\Omega]$} \\
$\delta_{0}$ & 0.085 & {$[\mathrm{~mm}]$} \\
$g$ & 9.81 & {$\left[\mathrm{~ms}^{-2}\right]$} \\
\hline$\mu_{0}$ & $4 \pi \times 10^{-7}$ & {$\left[\mathrm{Hm}^{-1}\right]$} \\
\hline
\end{tabular}
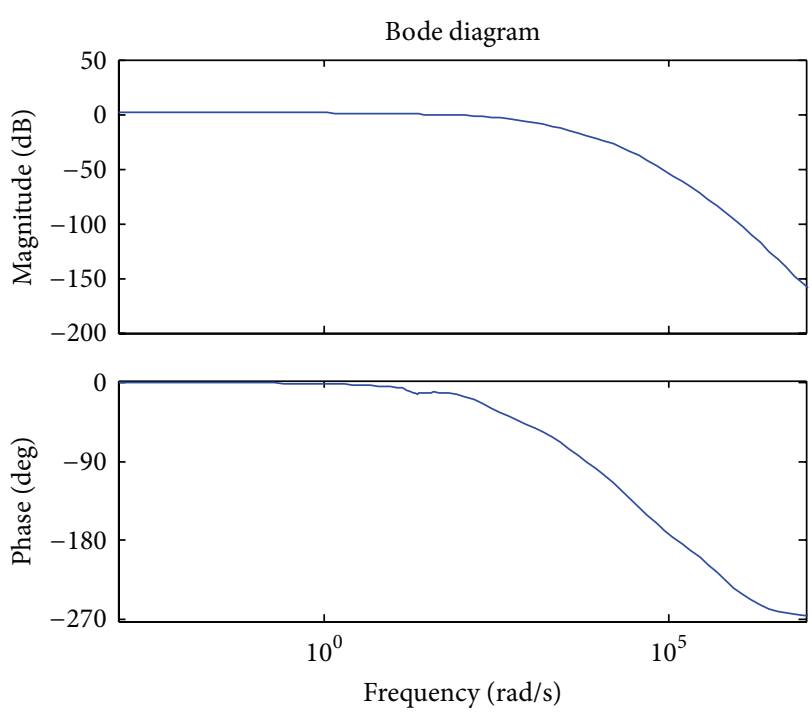

FIGURE 4: Closed-loop system Bode diagram with fractional order controller.

The controller design based on fractional order theory is according to Section 3. And the designed controller can be expressed as $C(s)=11940\left(1+0.1241 s^{0.851}\right)$. Figure 4 is the closed-loop system Bode diagram with fractional order controller.

From Figure 4, it is easy to know that the overshoot of the closed-loop system is very small. Bandwidth is about $15 \mathrm{~Hz}$, which can meet the requirement of the system. So, for these principles, the controller design based on fractional order theory can satisfy the basic demand of the maglev system.

4.2. Simulation Comparisons. This part compared the performance of the closed-loop system with different controllers under different working conditions.

At first, this part compared the step response of the two closed-loop systems. In the step response, the overshoot and rising time are the crucial factors we were concerned about. Figures 5 and 6 showed the step responses of the closed-loop system with controller based on $L Q R$ and controller based on fractional order theory, respectively.

Secondly, considering that the load of maglev suspension system is varying when operating, we hope the system can get stable performance when load is varying seriously. So, this section compared the step responses of the systems while 


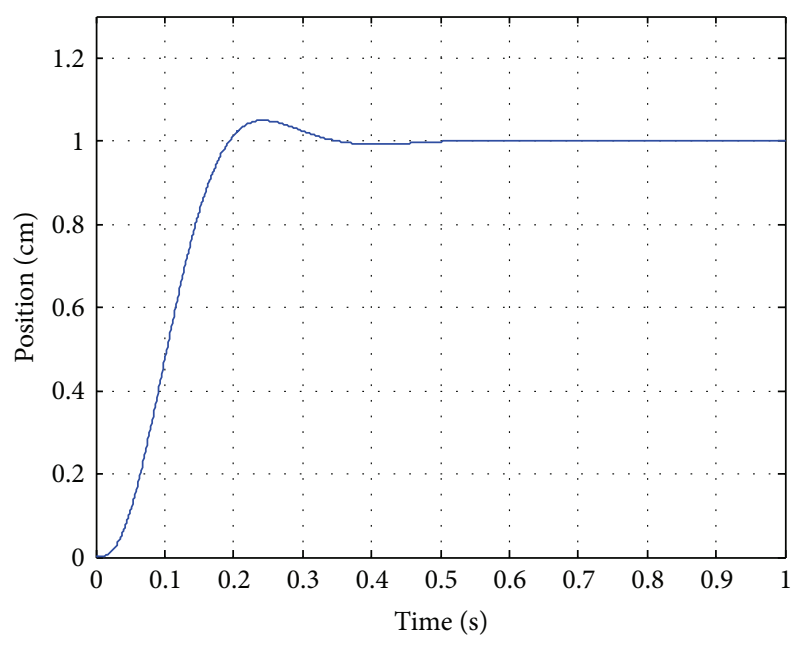

FIGURE 5: Step response of closed-loop system with $L Q R$ controller.

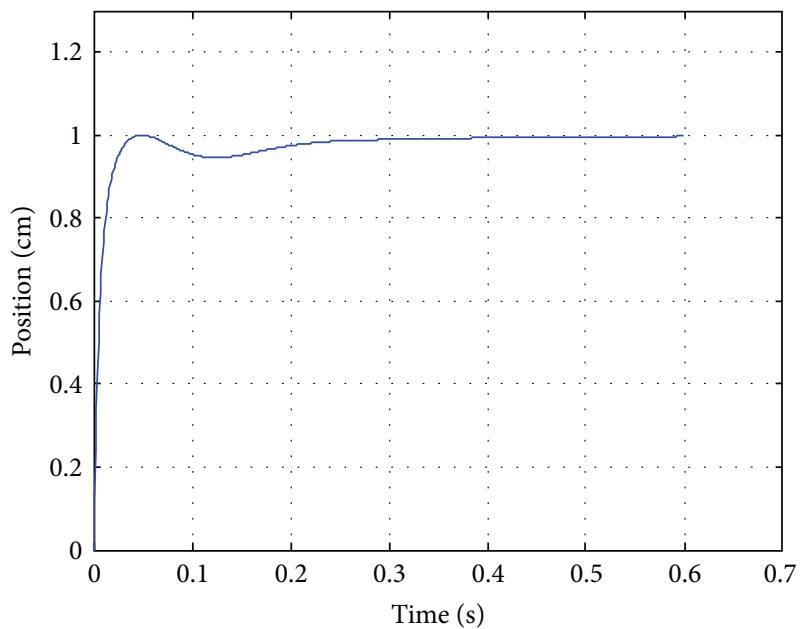

FIGURE 6: Step response of the closed-loop system with fractional order controller.

load is changing. Figures 7 and 8 show the step response of different controllers when the load $m$ is varying.

At last, when maglev train is running, the working gap is not constant. It is easy to know that when working position is varying, nearly all the parameters of the system will change. This phenomenon will bring the uncertain to the system and degrade the performance of the controller. So, much attention is paid to this problem. Figures 9 and 10 showed the step response of different controllers under different work positions.

In Figure 5, the rising time is about $0.18 \mathrm{~s}$ and the overshoot is about $5 \%$. In Figure 6, the rising time is $0.05 \mathrm{~s}$, much shorter than in Figure 5, and the overshoot can nearly be neglected. Comparing Figures 5 and 6, obviously, the system with fractional order controller has better performance, not only in overshoot, but also in rising time and settling time. The fractional order controller, with the characteristic of nonlinear, improved the system performance of rising time.

In Figure 7, when the load $m$ changes from $350 \mathrm{~kg}$ to $1250 \mathrm{~kg}$, not only the rising time, but also the overshoot of the

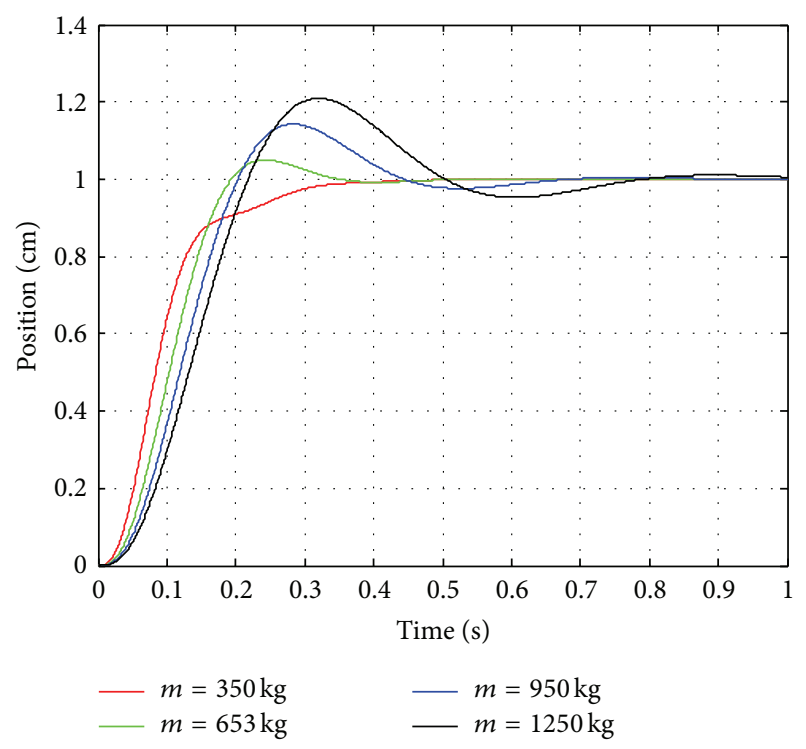

FIGURE 7: Step response of the closed-loop system with $L Q R$ controller when load is varying.

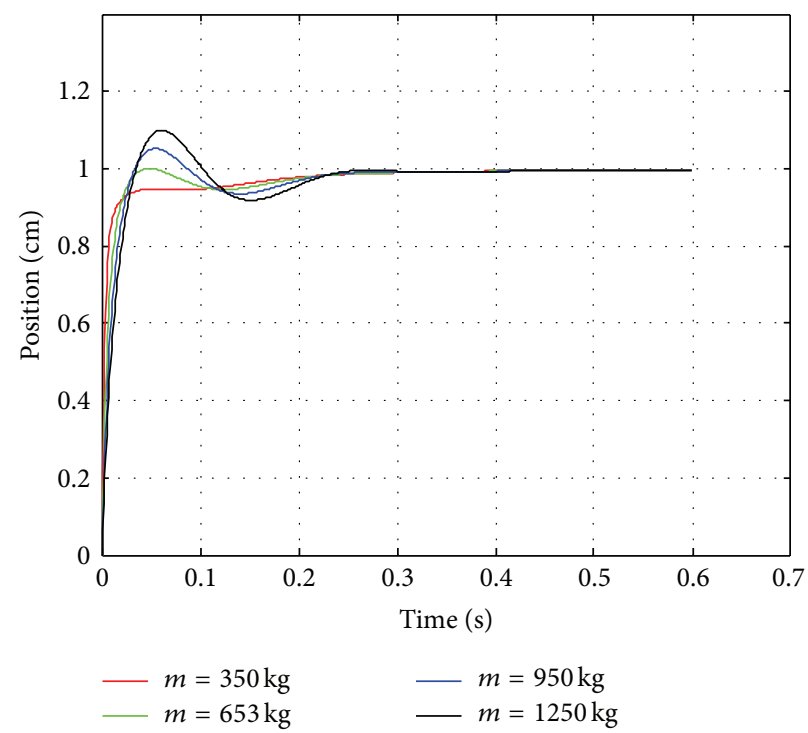

FIGURE 8: Step response of the closed-loop system with fractional order controller when load is varying.

step response varies seriously. And the heavier the load is, the longer the rising time is and the larger the overshoot is. When $m=1250 \mathrm{~kg}$, compared with $m=650 \mathrm{~kg}$, the overshoot is more than $20 \%$. The rising time, compared with $m=653 \mathrm{~kg}$, is about $0.08 \mathrm{~s}$ longer. Similar variables are applied to the system with fractional order controller and Figure 8 shows the response of it.

In Figure 8 , the overshoots are smaller than those in Figure 7. When $m=1250 \mathrm{~kg}$, the overshoot is less than $10 \%$, and the rising time is less than $0.05 \mathrm{~s}$. When $m$ changed to $653 \mathrm{~kg}$, the overshoot and rising time are varying, but they are smaller than the response controlled by $L Q R$ controller all the same. 


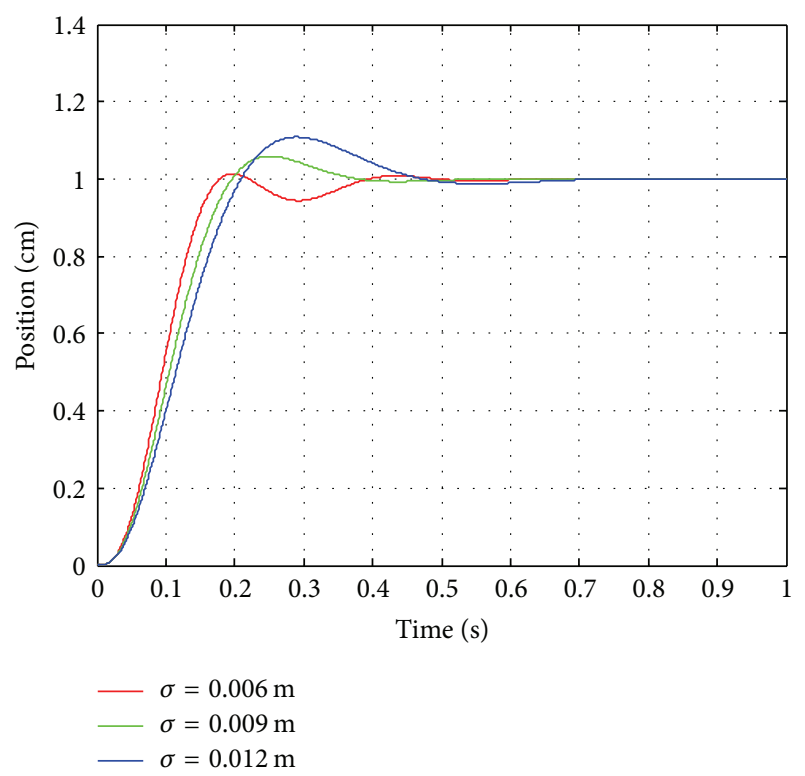

FIGURE 9: Step response of the closed-loop system with $L Q R$ controller when working position is varying.

Figures 7 and 8 present the step response when the load $m$ is varying. In contrast, in Figure 8, although the load $m$ is varying as in Figure 7, the overshoot and rising time of the step response are changing much smaller than in Figure 7. That means the system with fractional order controller is robust to load varying.

There is also a need to pay attention to the fact that, in Figure 8 , when $m$ is varying, the difference of the response exists.

In Figure 9, when the working position changes from $0.006 \mathrm{~m}$ to $0.012 \mathrm{~m}$, the rising time and the overshoot of the step response varied with the changing of working position.

Figures 9 and 10 are simulating for the different operating positions with different controllers.

In Figure 10, the overshoot and rising time remain constant when working position is varying. On the other hand, in Figure 9, the rising time and overshoot are unstable and changing seriously. The system performance with $L Q R$ controller is sensitive to the varying of working position. But, in Figure 10, it is obvious that system with fractional order controller is not sensitive to the change of working position and is robust to this kind of parameter change.

The conclusion can be concluded that the fractional order controller can suppress the disturbance brought by working position varying remarkably.

\section{Conclusions}

(1) This paper applied a fractional order controller on the maglev suspension system and introduced the procedure of the controller design. Three specifications are discussed for the selection of parameters of the controller.

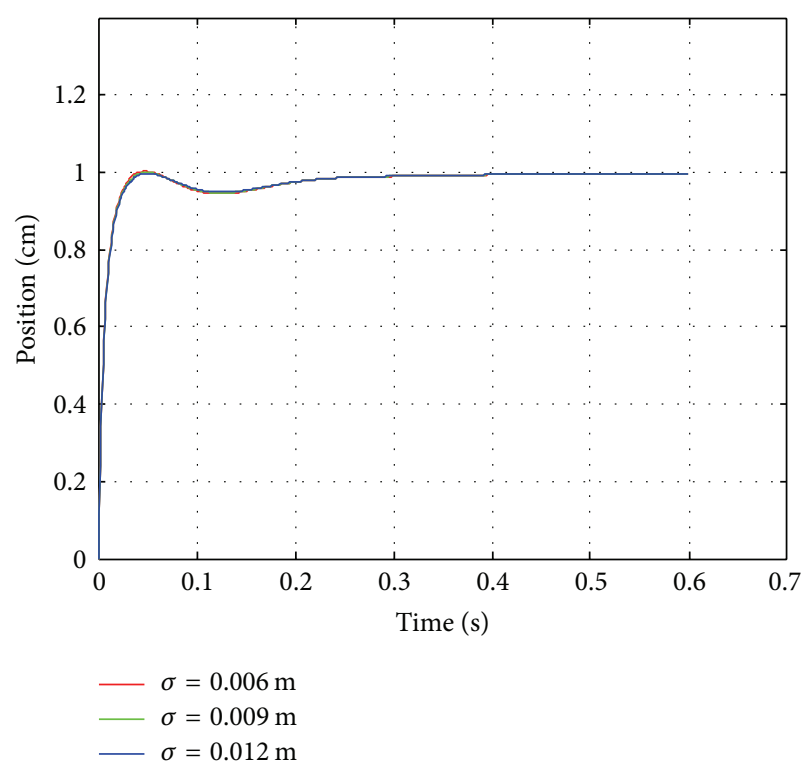

FIGURE 10: Step response of the closed-loop system with fractional order controller when work position is varying.

(2) Thanks to its nonlinear feature and higher degree free, the fractional order controller, compared with classical controller based on $L Q R$, can achieve better dynamic performance and robustness for the system, especially to the uncertain load and operating gap. Simulation results show the advantages of the fractional order controller.

(3) In the next stage, the main work should be focused on the realization of the controller on hardware for the usage in practical suspension system and the building of a reliable fractional order model for the suspension system.

\section{Conflict of Interests}

The authors declare that there is no conflict of interests regarding the publication of this paper.

\section{Acknowledgment}

The authors gratefully acknowledge financial support supported by the National Natural Science Foundation of China (no. 11202230 and no. 11302252).

\section{References}

[1] P. K. Sinha, Electromagnetic Suspension: Dynamics \& Control, Short Run Press, London, UK, 1987.

[2] L.-G. Yan, "Development and application of the Maglev transportation system," IEEE Transactions on Applied Superconductivity, vol. 18, no. 2, pp. 92-99, 2008.

[3] H.-B. Zhou and J.-A. Duan, "Levitation mechanism modelling for maglev transportation system," Journal of Central South University of Technology, vol. 17, no. 6, pp. 1230-1237, 2010. 
[4] L. Zhang, S. A. Campbell, and L. Huang, "Nonlinear analysis of a maglev system with time-delayed feedback control," Physica D: Nonlinear Phenomena, vol. 240, no. 21, pp. 1761-1770, 2011.

[5] T.-S. Yoon, F.-G. Wang, S.-K. Park, G.-P. Kwak, and H.-K. Ahn, "Linearization of T-S fuzzy systems and robust $H_{\infty}$ control," Journal of Central South University of Technology, vol. 18, no. 1, pp. 140-145, 2011.

[6] L. Yung, D. Zhang, and C. Hu, "The design and simulation of an adaptive maglev control algorithm based on oscillation observation," in Proceedings of the 18th International Conference on Magnetically Levitated Systems and Linear Drives (MAGLEV '04), vol. 2, pp. 984-990, October 2004.

[7] S. Westerlund and L. Ekstam, "Capacitor theory," IEEE Transactions on Dielectrics and Electrical Insulation, vol. 1, no. 5, pp. 826-839, 1994.

[8] Three-parameter tunable tilt integral derivative (TID) controller, US Patent US5371670, 1994.

[9] A. Oustaloup, J. Sabatier, and P. Lanusse, "From fractal robustness to the CRONE control," Fractional Calculus and Applied Analysis, vol. 2, no. 1, pp. 1-30, 1999.

[10] I. Podlubny, Fractional Differential Equations, Academic Press, 1999.

[11] Y. Q. Chen, H.-S. Ahn, and I. Podlubny, "Robust stability check of fractional order linear time invariant systems with interval uncertainties," Signal Processing, vol. 86, no. 10, pp. 2611-2618, 2006.

[12] I. Podlubny, "Fractional-order systems and $P I^{\lambda} D^{\mu}$ controllers," IEEE Transactions on Automatic Control, vol. 44, no. 1, pp. 208214, 1999.

[13] J. Yang, A. Zolotas, W.-H. Chen, K. Michail, and S. Li, "Robust control of nonlinear MAGLEV suspension system with mismatched uncertainties via DOBC approach," ISA Transactions, vol. 50, no. 3, pp. 389-396, 2011.

[14] Y. Luo and Y. Chen, "Fractional order [proportional derivative] controller for a class of fractional order systems," Automatica, vol. 45, no. 10, pp. 2446-2450, 2009.

[15] Y. Luo, Y. Q. Chen, H.-S. Ahn, and Y. G. Pi, "Fractional order robust control for cogging effect compensation in $\mathrm{PM} / \mathrm{SM}$ position servo systems: Stability analysis and experiments," Control Engineering Practice, vol. 18, no. 9, pp. 1022-1036, 2010.

[16] M. Caputo and F. Mainardi, "A new dissipation model based on memory mechanism," Pure and Applied Geophysics, vol. 91, no. 1, pp. 134-147, 1971.

[17] A. Oustaloup, F. Levron, B. Mathieu, and F. M. Nanot, "Frequency-band complex noninteger differentiator: characterization and synthesis," IEEE Transactions on Circuits and Systems I: Fundamental Theory and Applications, vol. 47, no. 1, pp. 25-39, 2000. 


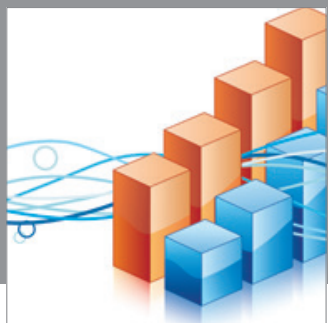

Advances in

Operations Research

mansans

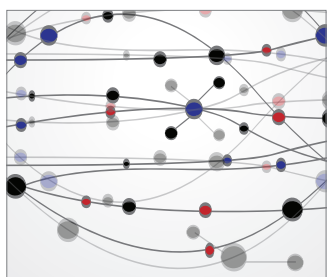

The Scientific World Journal
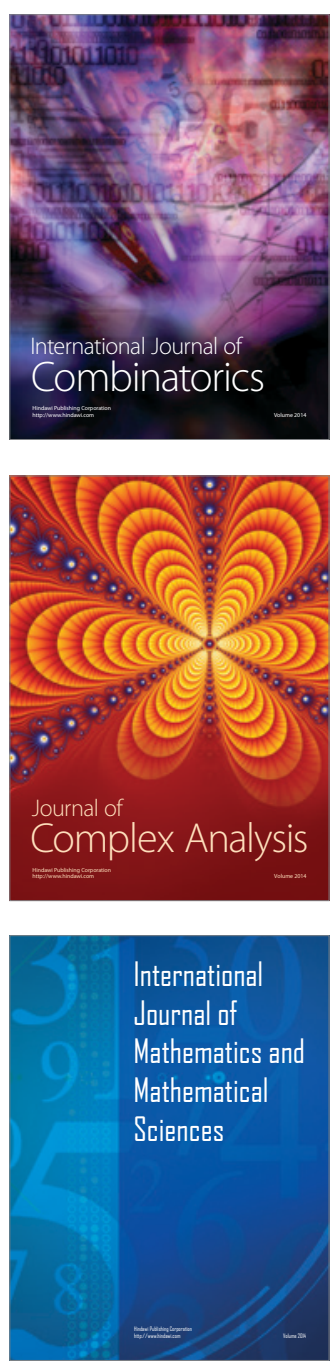
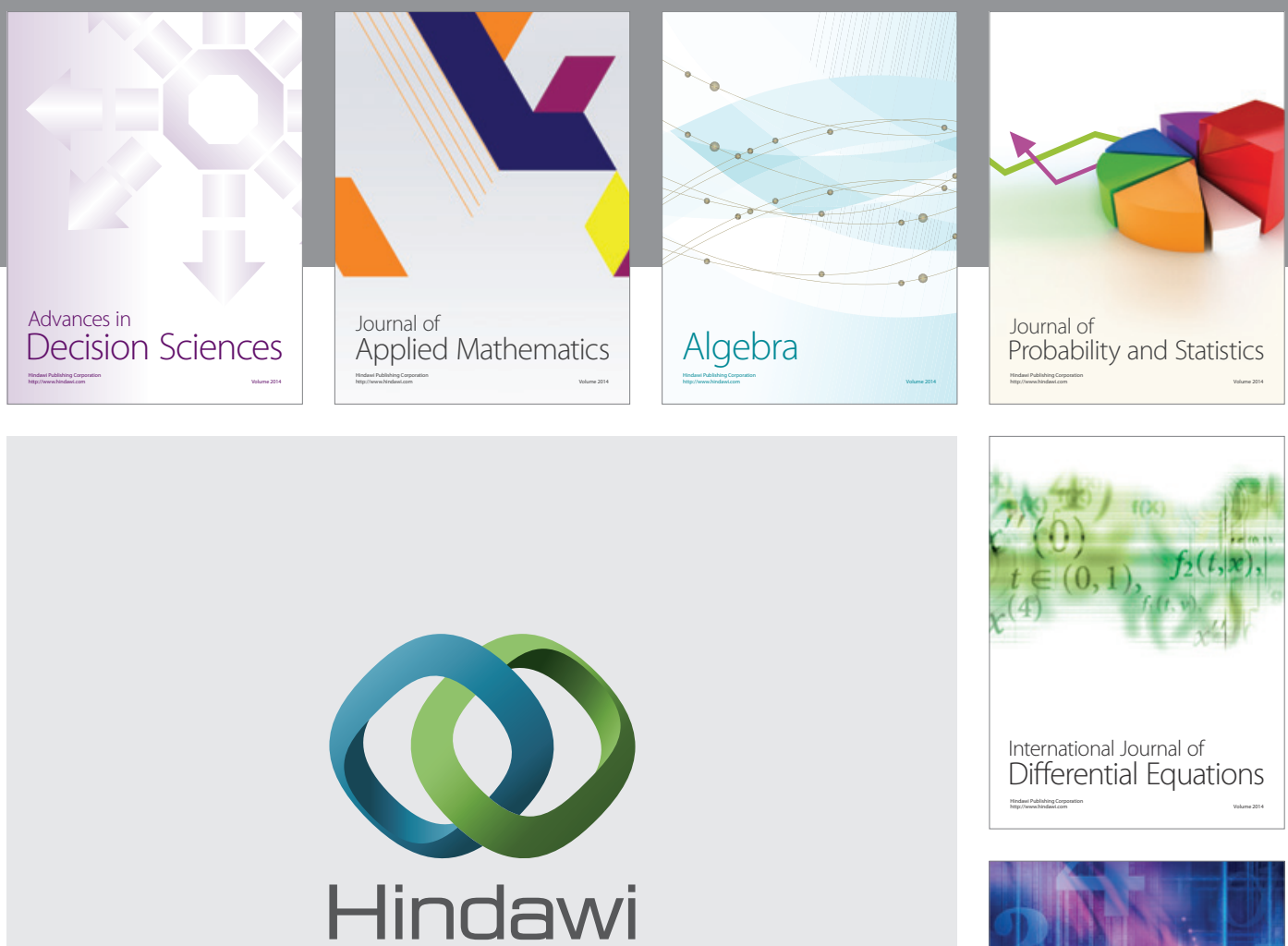

Submit your manuscripts at http://www.hindawi.com
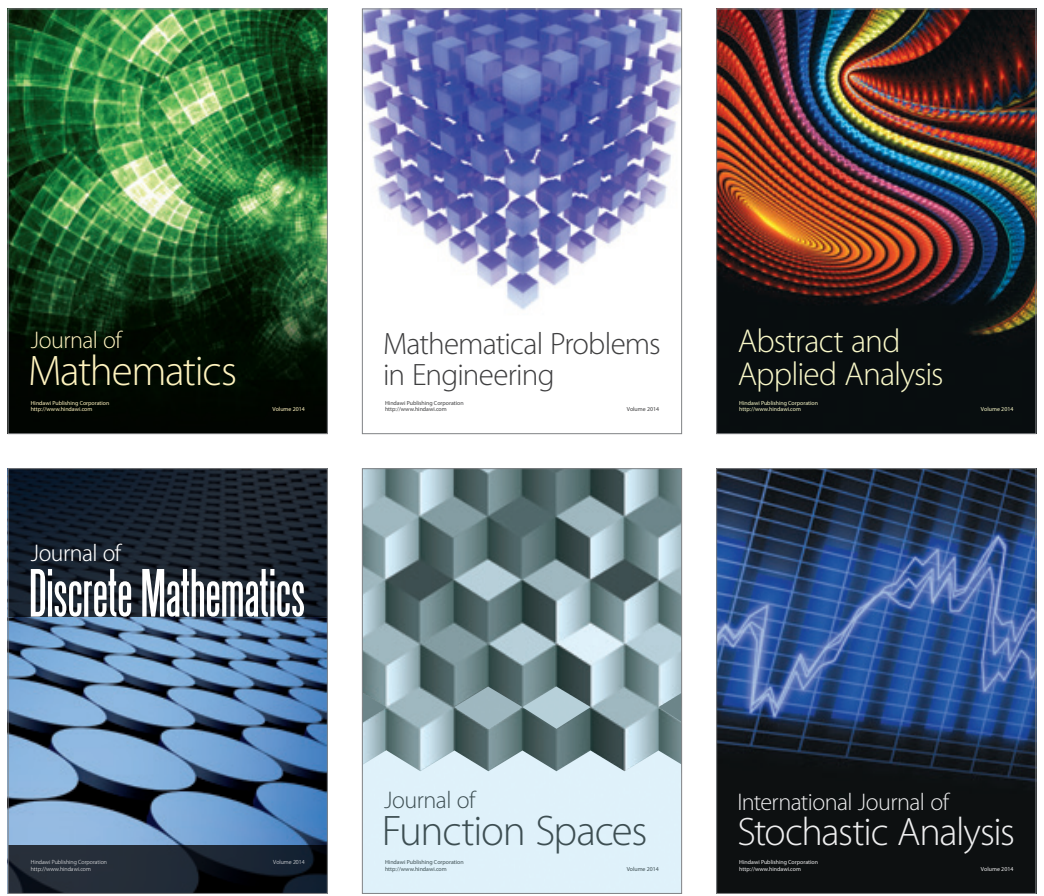

Journal of

Function Spaces

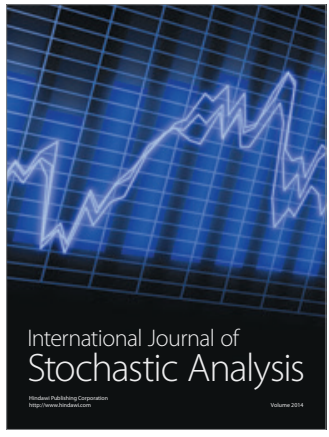

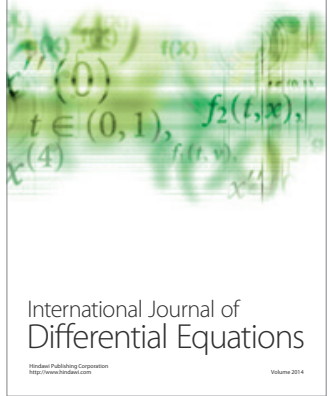
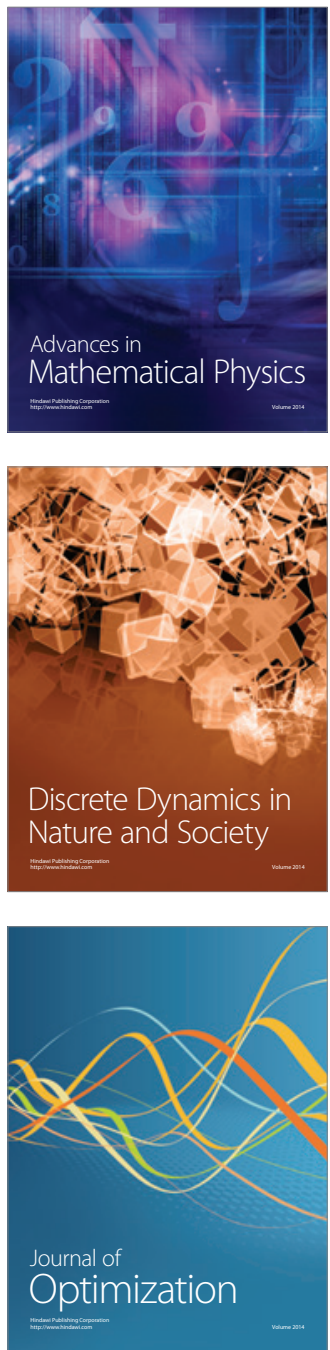